\title{
Test de germination des graines de Caesalpinia bonduc (L.) Roxb au Bénin
}

\author{
Comlan HESSOU, Romain GLELE KAKAÏ ${ }^{*}$, Achille E. ASSOGBADJO, \\ Théophile ODJO et Brice SINSIN
}

\author{
Laboratoire d'Ecologie Appliquée (LEA), Faculté des Sciences Agronomiques \\ Université d'Abomey-Calavi (UAC), 01 BP 526 Cotonou, Bénin \\ *Auteur correspondant, E-mail: gleleromain@yahoo.fr
}

\section{RESUME}

La présente étude a pour objectif d'évaluer la germination des graines de deux provenances (Ouèdo/Bénin et Atakpamè/Togo) de Caesalpinia bonduc sur différents types de substrats (terreau chauffé, terreau traité aux fongicide et insecticide-nématicide et terreau non traité). Pour chacune des provenances, deux lots de 30 graines étaient considérées, les graines du premier lot étant débarrassées de leur péricarpe (amande nue) et celles du deuxième lot étant laissées telles quelles. Pour chaque lot de graines, deux répétitions ont été appliquées suivant un dispositif complètement aléatoire. Le semis a été réalisé dans des sachets de polystyrène noirs remplis de substrat. Les paramètres considérés pour chacun des traitements sont: le temps mis après semis pour la germination de la première graine et le taux final de germination. Ces paramètres ont été soumis à l'analyse de variance pour comparer les traitements appliqués aux graines, les provenances et les substrats. Il ressort de l'étude que, de façon générale, les graines non traitées mettent plus de temps à germer (16,4 jours) que celles débarrassées de leur péricarpe (13,1 jours). Par ailleurs, la provenance Atakpamè du Togo a présenté des taux de germination relativement faibles pour l'amande nue. L'étude a aussi permis de montrer que les taux de germination les plus élevés sont observés sur les substrats chauffés et non traités $(41,9 \%)$. Les deux provenances garantissent, quel que soit le traitement des graines, un taux moyen de germination minimal compris entre 40 et $50 \%$. Les résultats obtenus de la présente étude permettent alors de conseiller la technique de l'amande nue pour la provenance Ouèdo du Bénin pour améliorer les pratiques traditionnelles de propagation de l'espèce à partir de ses graines.

(c) 2009 International Formulae Group. All rights reserved.

Mots-clés: Caesalpinia bonduc, propagation, provenances, substrats, Bénin, Togo.

\section{INTRODUCTION}

Caesalpinia bonduc (L.) Roxb est un arbuste épineux natif de la Floride aux USA (Wunderlin, 1998). L'espèce a longtemps été confondue à Caesalpinia cristata Auct. (Akoègninou et al., 2006). Elle est rencontrée sur les littoraux, au niveau des plages, d'estuaires, et de rives de lagons (Boullard, 2001). Elle est distribuée dans toute la zone tropicale et sub-tropicale (Kinoshita, 2000). L'arbuste a une hauteur et un diamètre moyens respectivement de $1,5 \mathrm{~m}$ et $5 \mathrm{~cm}$; toutefois, il peut atteindre $5 \mathrm{~m}$ de hauteur; sa tige est monocaule mais assez bas-branchue
(Parrotta, 2001). Ses rameaux gris et duveteux portent des feuilles composées, paripennées, longues de 20 à $40 \mathrm{~cm}$ de long et regroupant 12 à 16 folioles oblongues (Harden, 2002). Les fruits sont des gousses comprimées et cornées, pourvues de nombreuses épines courtes; ils contiennent 1 à 4 graines dures, coriaces mais légères, ovoïdes et bleuâtres, grosses comme des billes. La graine présente un péricarpe assez dur et présente en moyenne 409 graines $/ \mathrm{kg}$, soit un poids moyen par graine de 2,44 \pm 0,03 g (Nelson, 1996).

Le bois dur et rouge foncé est utilisé en tannerie. Il a remplacé avantageusement le 
sappan (Caesalpinia sappan), pour sa teinture. Il est extrait des graines une huile résineuse, efficace en usage externe, contre les convulsions, les paralysies et la blennorragie. La tisane de racines est vermifuge, diurétique fébrifuge et fait diminuer les règles abondantes (Boullard, 2001). Des extraits de la graine combattraient aussi le stress et augmenteraient l'endurance durant la natation (Kannur et al., 2006). Kerharo et Adam (1974) ont rapporté l'existence de substances chimiques pharmaceutiques dans la graine (la bonducine) et dans la tige (la brasiline et la brasilène). Par ailleurs, tous les organes de la plante sont utilisés à diverses fins en zone tropicale (Kinoshita, 2000). Au Bénin, la graine intervient en géomancie et est aussi utilisée dans le jeu de domino appelé «Adji » en langue fon au Bénin ou «Awale» sur le plan international. Ses racines sont utilisées dans le traitement de l'adénome prostatique (de Souza, 2006). C'est ce dernier usage très répandu au Bénin qui constitue la plus grande pression sur la survie de l'espèce. De plus, les graines sont très dures et ne favorisent pas toujours la régénération spontanée de l'espèce dans la nature. Les différentes pressions anthropiques sur $C$. bonduc combinées aux difficultés de régénération lui confèrent le statut d'espèce menacée (Baillie et al., 2004). De ce fait, la maîtrise des techniques de multiplication végétative de cette espèce est d'une grande importance pour sa conservation.

Nous proposons dans la présente étude d'explorer les aptitudes des graines de $C$. bonduc à la germination. Par ailleurs, les graines ayant un péricarpe dur comme $C$. bonduc nécessitent l'application des méthodes de scarification mécanique (Teketay, 1996; Bationo et al., 2001; Razanameharizaka et al., 2006).

La provenance des graines est aussi un facteur susceptible d'influencer leur pouvoir germinatif (Assogbadjo, 2006). Nous avons donc évalué l'effet de la scarification sur les graines de deux provenances (Ouédo/Bénin et Atakpamè/Togo) en nous basant sur l'hypothèse que le taux de germination des graines de $C$. bonduc varie en fonction de leur zone de provenance, de la présence ou non de leur péricarpe et/ou en fonction du type de substrat utilisé. Ainsi, les objectifs de la présente étude étaient d'évaluer l'influence de la zone de provenance, de la scarification mécanique du tégument externe des graines et du substrat utilisé sur la germination des graines de $C$. bonduc.

\section{METHODES}

\section{Milieu d'étude}

Le site d'expérimentation considéré (Abomey-Calavi) est situé dans le domaine guinéen à $15 \mathrm{~km}$ au Nord de Cotonou (6 $30^{\circ}$ Nord et $\left.2^{\circ} 24^{\prime} \mathrm{E}\right)$. Il présente un climat de type subéquatorial avec un régime pluviométrique bimodal à deux saisons humides (avril à juillet et octobre à novembre) intercalées par deux saisons sèches (août à septembre et décembre à mars). La pluviométrie annuelle varie de 1100 à 1300 $\mathrm{mm}$, tandis que la température moyenne annuelle est de l'ordre de $25{ }^{\circ} \mathrm{C}$ avec une amplitude de $4{ }^{\circ} \mathrm{C}$. Les maxima de température sont souvent enregistrés en mars tandis que les minima sont généralement enregistrés en août. Quant à l'humidité relative, elle atteint 96 à $97 \%$ en fin de saison pluvieuse (juin-juillet et novembre) et 34 à $36 \%$ en fin de saison sèche (janvier-février) soit une moyenne annuelle de 74,2\%. De décembre à février, l'harmattan un air continental sec se manifeste durant quelques jours (Adam et Boko, 1993).

\section{Dispositif expérimental et collecte des données}

Les semences de la provenance Ouèdo (Bénin) ont été récoltées en décembre 2005 à $29 \mathrm{~km}$ au Nord-Ouest de Cotonou. Celles de la provenance Atakpamè (Togo) ont été aussi récoltées en décembre 2005 dans la localité d'Atakpamè au Centre du Togo. La conservation des graines des deux provenances a été faite à l'air libre pendant 4 mois.

La différence entre les provenances est essentiellement d'ordre morphologique comme l'indique le tableau 1. Les semis ont eu lieu le 03 mars 2006, à la ferme de l'Université d'Abomey-Calavi, à raison d'une graine par sachet de $8 \mathrm{~cm}$ de diamètre et de $18,5 \mathrm{~cm}$ de hauteur chacun, suivant un dispositif complètement aléatoire, avec deux répétitions de 30 graines par combinaison des niveaux des facteurs.

Les facteurs contrôlés dans l'expérimentation étaient les provenances à 
Tableau 1: Quelques caractéristiques morphologiques des provenances.

\begin{tabular}{lccc}
\hline Provenances & $\begin{array}{c}\text { Nombre de } \\
\text { graines/kg }\end{array}$ & $\begin{array}{c}\text { Forme des } \\
\text { graines }\end{array}$ & Taille des foliolules \\
\hline $\begin{array}{l}\text { Ouèdo } \\
\text { (Benin) }\end{array}$ & 371 & $\begin{array}{c}\text { Oblongue } \\
\text { avec un méplat }\end{array}$ & $\begin{array}{c}2,3 \text { à } 5,2 \mathrm{~cm} \text { de long et } \\
1,5 \text { à } 2,5 \mathrm{~cm} \text { de large }\end{array}$ \\
$\begin{array}{l}\text { Atakpamè } \\
\text { (Togo) }\end{array}$ & 410 & $\begin{array}{c}\text { Même forme } \\
\text { que Ouèdo }\end{array}$ \\
\hline
\end{tabular}

savoir Ouèdo/Bénin et Atakpamè/Togo, les traitements effectués sur les graines à savoir, la graine entière (T1) et l'amande nue (T2) et les substrats considérés (terreau chauffé à 90 ${ }^{\circ} \mathrm{C}$ au moins pendant 30 minutes, terreau traité aux produits chimiques: fongicide à $0,5 \mathrm{~kg}$ de Manate 80; insecticide-nématicide à $1 \mathrm{~kg}$ de Fura $3 \mathrm{G}$, terreau non traité). Ces produits chimiques ont été appliqués 48 heures avant le semis. Le terreau provient d'un site de dépôt et de gestion des déchets ménagers à Hêvié, un village situé à proximité de Ouèdo. Il a ensuite été tamisé avant usage.

Les pots ont été arrosés jusqu'à ce que le terreau soit à sa capacité au champ. Cela correspond à un dosage sans aucune perte d'eau à $235 \mathrm{ml} /$ pot. Mais en pratique, avec le système courant d'arrosage manuel, cela nécessite 300 litres d'eau par planche de 1000 pots. Par la suite, deux arrosages plus légers ont eu lieu chaque jour, le matin entre 7 et 8 heures et l'après-midi entre 16 et 17 heures GMT comme recommandé par Aho et Kossou (1997).

Les observations effectuées chaque jour, portent sur la date de première germination dans chaque lot de 30 graines et le nombre de graines germées pour chaque traitement, sur une durée de 30 jours.

\section{Traitement des données}

Les paramètres de comparaison des niveaux des 3 facteurs considérés sont le taux de germination et le nombre de jours de première germination après semis. Le taux de germination ( $\mathrm{Tg}$ ) a été calculé quotidiennement durant les 30 jours d'expérimentation suivant la formule:

$\operatorname{Tg}=100 * \frac{\text { Nombre de semis constatés }}{\text { Nombre de graines semées }}$
Le nombre de jours de première germination est noté depuis la date du semis jusqu'au jour où la première graine a germé.

Les données liées au nombre de jours de première germination et au taux de germination ont d'abord subi une transformation logarithmique de base 10 . Elles ont été ensuite soumises à l'analyse de la variance à trois facteurs de classification, modèle fixe, afin de tester la différence entre les niveaux des trois facteurs au risque de $5 \%$. La transformation logarithmique a été appliquée aux données pour assurer la normalité et l'homogénéité des variances, nécessaires pour l'application de l'analyse de la variance. Par ailleurs, le test de StudentNewman-Keuls (Dagnelie, 1998) a été appliqué pour hiérarchiser les moyennes en cas de différences significatives entre les niveaux des facteurs. Le logiciel SASv9 (SAS, 2003) a été utilisé à cet effet. Par ailleurs, des diagrammes d'interaction ont été construits pour mieux décrire les interactions significatives entre facteurs.

\section{RESULTATS}

Les résultats des analyses de variance sur les nombres de jours de germination après semis et des taux de germination sont présentés au tableau 2. Il en ressort qu'aucune différence significative au seuil de 5\% n'est observée entre les trois substrats, ni entre les deux provenances de façon générale, en ce qui concerne le nombre de jours de première germination après semis. Par contre, il existe une différence significative au seuil de 5\% entre les graines débarrassées de leur péricarpe et celles non traitées. On note également une interaction entre la provenance et le traitement des graines. En ce qui concerne le taux de germination, on note au contraire une différence significative entre les 
Tableau 2 : Résultats d'analyse de variance.

\begin{tabular}{lccc}
\hline \multirow{2}{*}{ Sources de variation } & ddl & \multicolumn{2}{c}{ Probabilité } \\
\cline { 3 - 4 } & 2 & $\begin{array}{c}\text { Nombre de jours } \\
\text { après semis }\end{array}$ & $\begin{array}{c}\text { taux de } \\
\text { germination }\end{array}$ \\
\hline Substrats & 1 & $0,370 \mathrm{~ns}$ & $0,048 *$ \\
Provenances & 1 & $0,120 \mathrm{~ns}$ & $0,026 *$ \\
Traitements & 2 & $0,010 * *$ & $0,29 \mathrm{~ns}$ \\
Substrats*Provenances & 2 & $0,430 \mathrm{~ns}$ & $0,92 \mathrm{~ns}$ \\
Substrats*Traitements & 1 & $0,000 * * *$ & $0,96 \mathrm{~ns}$ \\
Provenances *Traitements & 2 & $0,870 \mathrm{~ns}$ & $0,000 * * *$ \\
Substrats*Provenances*Traitements & 12 & - & $0,018 *$ \\
Erreurs & 23 & - & - \\
Total & 23 & - \\
\hline ns : différence non significative au seuil de $5 \% ; *:$ différence significative au seuil de $5 \% ; * *:$ différence \\
significative au seuil de $1 \% ; * * *:$ différence significative au seuil de $0,1 \%$.
\end{tabular}

substrats et entre les provenances. Par contre, aucune différence n'est notée entre les traitements. Les interactions entre provenances et traitements d'une part et entre substrats, provenances et traitements d'autre part, sont significatives pour le taux de germination.

L'étude de l'interaction provenance* traitement montre que, les graines de Ouèdo scarifiées germent plus rapidement (8 jours après semis) que les non scarifiées de Ouèdo et celles de Atakpamè scarifiées ou non (Figure 1). Il faut remarquer que chez les graines de provenance Atakpamè (Togo), celles qui sont scarifiées mettent plus de temps à germer que celles qui ne le sont pas. Ceci est exactement le contraire des graines provenant de Ouèdo. On note de façon générale que, lorsqu'on considère tous les substrats et toutes les provenances, l'amande nue germe plus rapidement $(13,1 \pm 2,1$ jours $)$ que la graine non scarifiée (16,4 $\pm 3,2$ jours). Par ailleurs, quels que soient la provenance et le traitement, le nombre moyen de jours que mettent les graines pour germer après semis est compris entre 14 et 16 .

Le tableau 3 présente les taux moyens de germination sur les différents substrats. Son analyse révèle que les substrats chauffés et non traités présentent pratiquement le même taux moyen de germination $(41,9 \%)$. Le substrat traité chimiquement présente un taux moyen de germination relativement faible $(41,3 \%)$.
L'étude de l'interaction montre ici que le taux moyen de germination est plus élevé au sein des graines de $C$. bonduc d'origine togolaise non scarifiée et chez celle de Ouèdo (Bénin) scarifiée que les autres combinaisons (Figure 2). On remarque aussi que les graines provenant du Togo germent plus vite sans aucune scarification de leur péricarpe contrairement à celles de Ouèdo qui germent plus vite après scarification. Mais il faut noter pour ce paramètre que le type de substrat influence l'effet combiné de la provenance et du traitement des graines à cause de l'interaction significative entre les trois facteurs. Dans tous les cas, on déduit de la figure 2 que les deux provenances garantissent, quel que soit le traitement des graines, un taux moyen de germination compris entre 40 et $50 \%$.

La figure 3 présente le nombre moyen de jours après semis nécessaires à la germination des graines (NBJAS) et le taux moyen de germination pour les différentes combinaisons des niveaux des trois facteurs. On y note que les graines de Atakpamè (Togo) non scarifiées et celles de Ouèdo (Bénin) scarifiées donnent des résultats significativement semblables et un meilleur taux moyen de germination sur les trois substrats. Ces deux types de graines donnent des taux de germination plus importants sur le terreau non traité que sur celui traité chimiquement. Par contre, les graines de Togo scarifiées et celles de Ouèdo non scarifiées ont donné des taux moyens de germination plus 


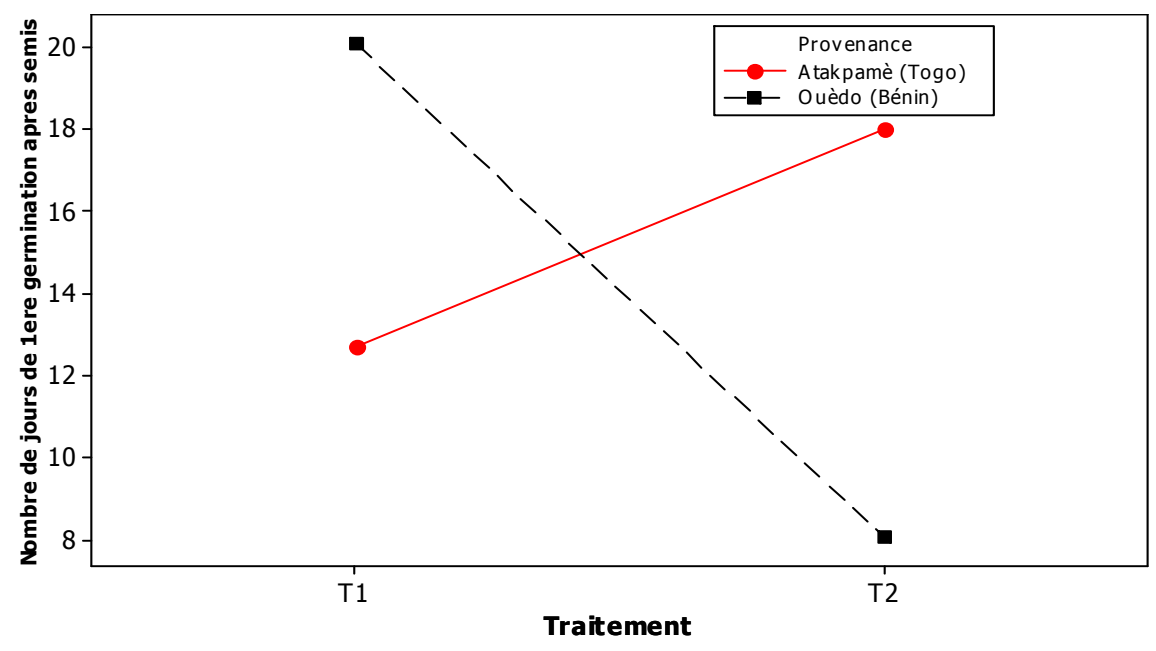

Figure 1: Diagramme d'interaction de l'effet combiné des traitements et provenances sur le taux moyen de germination. $\mathrm{T} 1=$ sans prétraitement; $\mathrm{T} 2$ : amande nue.

Tableau 3 : Taux moyen de germination de C. bonduc sur différents substrats.

\begin{tabular}{lcc}
\hline \multirow{2}{*}{ Substrats } & \multicolumn{2}{c}{ Taux moyen de germination } \\
& \multicolumn{2}{c}{$\mathbf{( \% )}$} \\
\cline { 2 - 3 } & $\mathbf{m}$ & $\mathbf{S}$ \\
\hline Terreau chauffé & $1,23(41,86) \mathrm{b}$ & 0,25 \\
Terreau traité chimiquement & $1,32(41,27) \mathrm{a}$ & 0,21 \\
Terreau non traité & $1,23(41,86) \mathrm{b}$ & 0,25 \\
\hline
\end{tabular}

Les moyennes suivies d'une même lettre ne sont pas significativement différentes; ( ): moyennes non transformées.

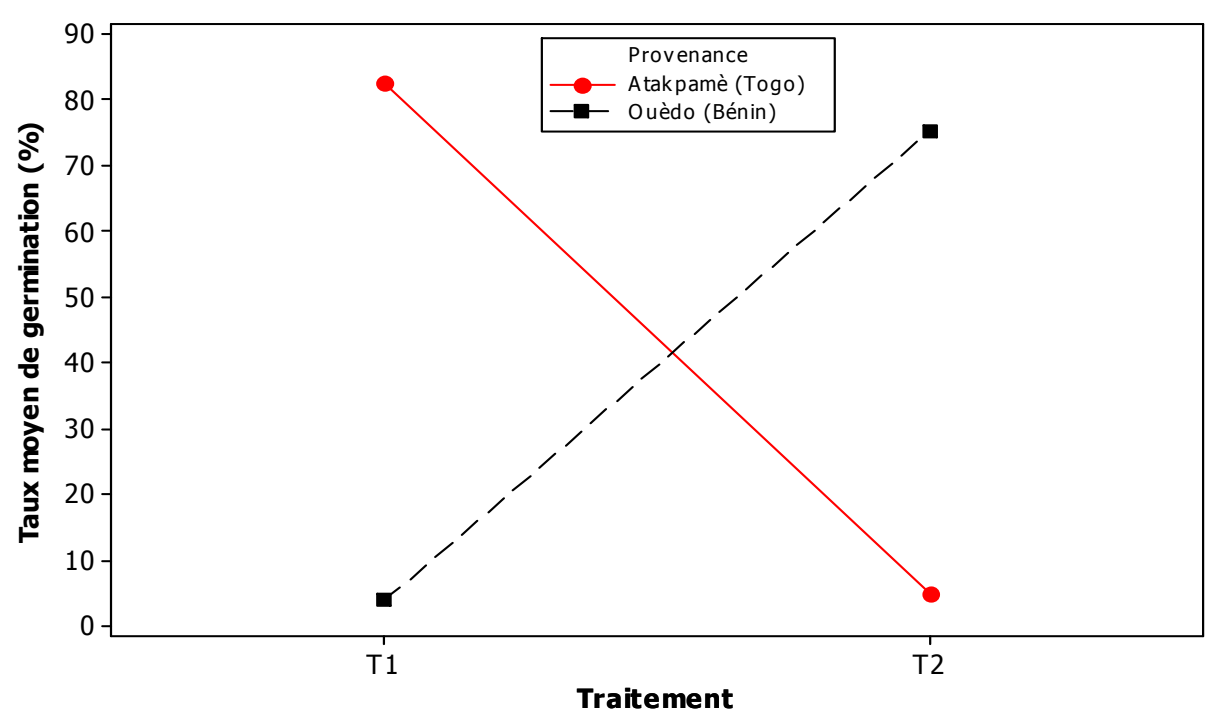

Figure 2 : Diagramme d'interaction de l'effet combiné des traitements et provenances sur le taux moyen de germination. $\mathrm{T} 1=$ sans aucun prétraitement $; \mathrm{T} 2:$ amande nue. 


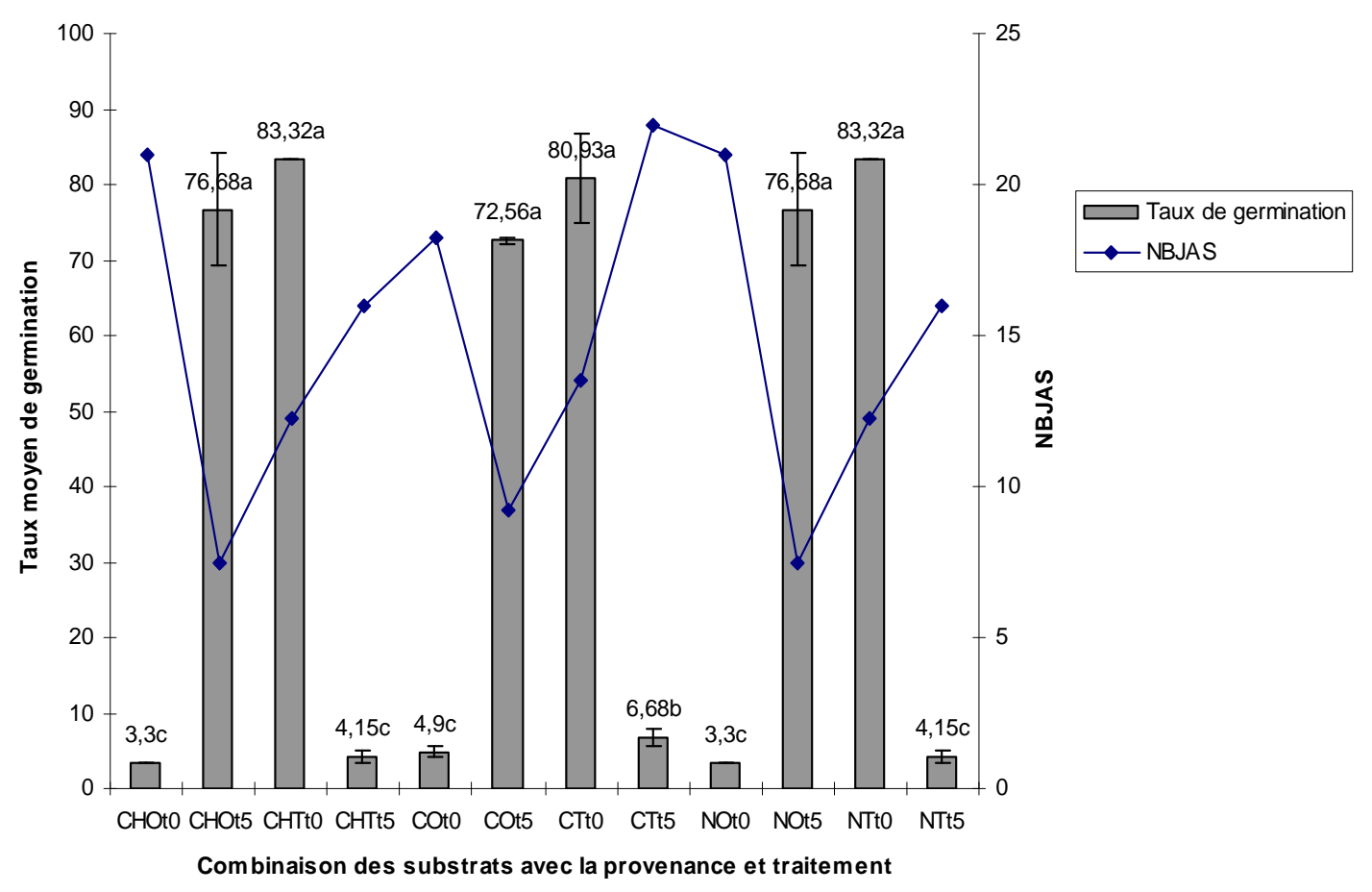

Figure 3: Dynamique de la germination: histogramme du taux moyen de germination en relation avec le nombre moyen de jours après semis. CHOt0: graine non scarifiée de Ouèdo sur substrat chauffé; CHOt5: graine scarifiée de Ouèdo sur substrat chauffé; CHTt0: graine non scarifiée du Togo sur substrat chauffé ; CHTt5: graine scarifiée du Togo sur substrat chauffé; COt0: graine non scarifiée de Ouèdo sur substrat traité chimiquement; COt5: graine scarifiée de Ouèdo sur substrat traité chimiquement; CTt0: graine non scarifiée du Togo sur substrat traité chimiquement; CTt5: graine scarifiée du Togo sur substrat traité chimiquement ; NTt0 : graine non scarifiée de Ouèdo sur substrat non traité; NTt5: graine scarifiée de Ouèdo sur substrat non traité; NOt0: graine non scarifiée de Togo sur substrat non traité; NOt5: graine scarifiée de Togo sur substrat non traité. NBJAS: nombre de jours après semis pour la germination des graines.

faibles surtout sur des terreaux non traités et chauffés.

Par ailleurs, l'analyse simultanée des deux représentations permet de percevoir le dynamisme de la germination; c'est-à-dire, la combinaison substrat-provenance-traitement qui donne un meilleur taux moyen de germination et un nombre moyen de jours après semis plus court. On note essentiellement deux groupes de traitements. Il s'agit du groupe des traitements occasionnant un taux de germination élevé avec un nombre moyen de jours après semis relativement court (inférieur à 9 jours) (CHOt5, CHTt0, COt5, CTt0, NOt5 et NTt0) d'une part et du groupe des semences de faible taux de germination et un nombre moyen de jours après semis plus long (supérieur à 15 jours) (CHOt0, CHTt5, COt0, CTt5, NOt0 et NTt5) d'autre part. Dans le premier groupe, on remarque que les graines scarifiées provenant de Ouèdo sur substrat chauffé (CHOt5) et les graines non scarifiées provenant de Atakpamè (Togo) sur substrat chauffé (CHTt0) ont donné le même résultat que les graines (scarifiées ou non) des deux provenances mais sur substrat neutre (NOt5 et NTt0). Pour ces traitements (CHOt5 et NOt5), on a obtenu un taux moyen de germination et un nombre moyen de jours après semis élevés (76,7\% et 7,5 jours). En ce qui concerne les traitements CHTt0 et NTt0, le taux moyen de germination est de $83,3 \%$ et un nombre de jours après semis de 12,3.

\section{DISCUSSION}

L'effet du substrat, de la provenance et de la scarification des graines de $C$. bonduc sur leurs taux moyen de germination et nombre moyen de jours après semis pour la première germination a été évalué dans la présente étude. Les résultats obtenus ont 
permis de noter l'effet positif de la scarification sur la germination des graines de C. bonduc de provenance Ouèdo (Bénin). Des résultats similaires ont été auparavant notés par Nelson (1996) pour des graines provenant de Porto-Rico. En effet, il a noté que la plupart des graines fraîches scarifiées avaient germé en un mois et la germination de toutes les graines a duré une année. Pour les graines fraîches non scarifiées, il a également noté qu'en 16 mois, seules 23 graines ont germé. Des résultats similaires ont été aussi obtenus par Costin (1965) pour des graines de $C$. bonduc collectées dans l'Antarctique.

Contrairement aux résultats obtenus cidessus, pour les graines de provenance Atakpamè (Togo), l'enlèvement du péricarpe a plutôt eu un impact relativement négatif sur leur germination. Cela suggère donc un effet de l'état de l'embryon de la graine mais surtout de ses téguments, les conditions et la durée de conservation étant les mêmes pour les deux provenances. Il est utile de noter que la dureté du péricarpe des graines de $C$. bonduc leur permet de conserver leur pouvoir germinatif sur de longs termes. Markland (2002) a en effet noté que des graines de $C$. bonduc de 19 ans flottant sur l'eau en mer conservaient leur pouvoir germinatif. Par ailleurs, le taux de germination pour des graines de la plupart des espèces forestières semées immédiatement après récolte ne diffère pas significativement si les graines sont semées fraîches, après un séchage de 8 jours à l'air à la température ambiante (26-28 ${ }^{\circ} \mathrm{C}$ ), ou après 8 jours de séchage au soleil (30$\left.34{ }^{\circ} \mathrm{C}\right)$ (Ezumah, 1986). En revanche, les fruits frais cueillis ne donnent pas toujours la meilleure germination comme c'est le cas de Gmelina arborea dont les graines stockées pour une longue durée germaient mieux que celles fraîchement cueillies (Bowen et Eusebio, 1983).

En conditions naturelles, les facteurs de scarification des graines de C. bonduc sont les grains de sable, les insectes et les rongeurs qui favorisent la pénétration de l'eau dans les graines et ainsi leur germination (Markland, 2002). Par ailleurs, la différence de comportement notée dans la présente étude entre les deux provenances, en ce qui concerne l'effet du traitement des graines sur la germination, pourrait aussi être liée à une différenciation génétique entre les deux provenances. En effet, Dangasuk et al. (1997) ont montré pour 12 provenances africaines de Faidherbia albida, des variations génétiques liées aux traits des graines et qui ont été susceptibles d'influencer leur pouvoir germinatif.

Par ailleurs, l'absence de différence significative au niveau de l'interaction entre les facteurs 'substrats', 'provenances' et 'traitements', en ce qui concerne le nombre moyen de jours de première germination après semis montre bien que ce comportement des graines des deux provenances ne dépend pas d'un substrat donné. Cela peut se comprendre par le fait que le terreau non traité n'était pas forcément infecté au départ, ce qui explique qu'il n'y ait pas de différence avec les autres états de substrat.

En ce qui concerne l'effet des substrats sur la germination des graines, le taux moyen de germination relativement faible $(41,3 \%)$ noté pour le substrat traité chimiquement suggère une certaine inefficacité des produits chimiques utilisés, ou au contraire un effet d'intoxication dû aux produits. Par contre, sur les terreaux non traités et aussi chauffés, les graines d'Atakpamè (Togo) scarifiées et celles de Ouèdo (Bénin) non scarifiées ont présenté des taux moyens de germination plus faibles. Cela suppose encore une fois que le taux de germination des graines de Ouèdo non scarifiées n'est pas dépendant de l'état du substrat.

\section{Conclusion}

La présente étude confirme que la dormance des graines de $C$. bonduc est en partie d'ordre physique. C'est la coque qui fait obstacle à la germination de la graine, ce qui fait que le taux de germination, toutes choses égales par ailleurs, est plus élevé dès que le péricarpe est enlevé du moins pour la provenance Ouédo du Bénin. Aussi, le pouvoir germinatif de la graine dépend-il de sa provenance. Il ressort de tout ce qui précède que, la production de plantules de $C$. bonduc dans les pépinières doit utiliser des graines fraîchement extraites de leur capsule. De plus pour de telles graines, il est plus indiqué de scarifier leur péricarpe avant de les semer si la provenance Ouèdo du Bénin est considérée; dans le cas de l'utilisation de la provenance Atakpamè du Togo, il n'est pas conseillé de les scarifier. Ainsi, le protocole 
utilisant des graines fraîchement extraites de leur capsule et scarifiées avant semis (la méthode de l'amande nue) peut être conseillé aux forestiers et paysans qui veulent s'investir dans l'installation de pépinières de $C$. bonduc de provenance Ouèdo.

\section{REFERENCES}

Adam KS, Boko K. 1993. Le Bénin. Editions du Flamboyant: Cotonou.

Aho N, Kossou DK. 1997. Précis d'Agriculture Tropicale. Bases et Eléments d'Applications. Editions du Flamboyant: Cotonou.

Akoègninou A, Van Der Burg WJ, Van Der Maesen LJG, Adjakidje V J-P, Sinsin B, Yedomohan H. 2006. Flore Analytique du Bénin. Backhuys Publishers: Amsterdam.

Assogbadjo AE, Kyndt T, Sinsin B, Gheysen G, Van Damme P. 2006. Patterns of genetic and morphometric diversity in baobab (Adansonia digitata L.) populations across different climatic zones of Benin (West-Africa). Annals of botany, 97: 819-830.

Baillie JEM, Hilton-Taylor C, Stuart SN. 2004. 2004 IUCN Red List of Threatened Species. A Global Species Assessment. IUCN, Gland: Switzerland and Cambridge.

Bationo BA, Ouedraogo SJ, Guinko S. 2001. Longévité des graines et contraintes à la survie des plantules d'Afzelia africana Sm. dans une savane boisée du Burkina Faso. Ann. For. Sci., 58: 69-75.

Boullard B. 2001. Dictionnaire des Plantes Médecinales du Monde: Réalités et Croyances ( $1^{\text {ère }}$ édn). Estem: Paris.

Bowen MR, Eusebio TV. 1983. Gmelina arborea flowering and seed studies. Forest Genet. Res. Inform., 12: 27-28.

Costin AB. 1965. Long-distance seed dispersal to Macquarie Island. Nature, 206: 317-318.

Dagnelie P. 1998. Statistique Théorique et Appliquée (Vol. 2). De Boeck et Larcier: Paris.

Dangasuk OG, Gudu S, Okaebo JR. 1997. Early growth Performance of sixteen populations of Faidherbia albida in semi arid Baringo district of Kenya. In Sustaining Global Farm, Stott DE,
Mohtar RH, Steinhardt GC (éds). Purdue University press: Perdue; 412-418.

De Souza S. 2006. Le Domaine MédicoMagique et les Gris-Gris du Bénin. Editions Simone de Souza : Riez.

Harden GJ. 2002. Flora of NSW (Vol. 2, $2^{\text {nd }}$ edn). UNSW Press: Sydney.

Ezumah BS. 1986. Germination of storage of neem (Azadirachta indica A. Juss.). Seed Science and Technology, 14: 593-600.

Kannur DM, Hukkeri VI, Akki KS. 2006. Adaptogenic activity of Caesalpinia bonduc seed extracts in rats. Journal of Ethnopharmacology, 108(3): 327-331.

Kerharo J, Adam JG. 1974. La Pharmacopée Sénégalaise Traditionnelle-Plantes Médicinales et Toxiques. Ed. Vogot Frères: Paris.

Kinoshita T. 2000. Chemical studies on the Philippine Crude Drug Calumbibit (Seeds of Caesalpinia bonduc): The isolation of new cassane diterpenes fused with $\alpha, \beta$ Butenolide. Chem. Pharm. Bull., 48(9): 1375-1377.

Markland J. 2002. Drift seeds. In West Word, Community Paper for Glenfinnan, Lochailort, Glenuig, Arisaig, Morar, Mallaig, Knoydart, and the Small Isles. http://road-to-theisles.org.uk/westword/ march2002.html; 6-7

Nelson G. 1996. The Shrubs and Woody Vines of Florida. Pineapple Press, Inc: Sarasota.

Parrotta JA. 2001. Healing Plants of Pininsular India. CABI Publishing: Wellingford and New York.

Razanameharizaka J, Grouzis M, Ravelomanama D, Danthu P. 2006. Seed storage behaviour and seed germination in African and Malagasy baobabs (Adansonia species). Seed Science Research, 16: 83-88.

SAS. 2003. The SAS system for Windows; Version 9.1, SAS Institute Inc., Cary, NC.

Teketay D. 1996. Germination ecology of twelve indigenous and eight exotic multipurpose leguminous species from Ethiopia. Forest Ecology and Management, 80: 209-223.

Wunderlin RP. 1998. Guide to the Vascular Plants of Florida. Gainesville, Fl.: University Press: Florida. 